\title{
AV Block Second Degree Mobitz Type II
}

National Cancer Institute

\section{Source}

National Cancer Institute. AV Block Second Degree Mobitz Type II. NCI Thesaurus. Code C62018.

A disorder characterized by an electrocardiographic finding of intermittent failure of atrial electrical impulse conduction to the ventricles, characterized by a relatively constant PR interval prior to the block of an atrial impulse. (CDISC) 